\title{
The New Size-Biased Kumaraswamy-G Distribution
}

\author{
Clement Boateng Ampadu*
}

Department of Biostatistics, USA

*Corresponding author: Clement Boateng Ampadu, Department of Biostatistics, 31

\section{Abstract}

This paper introduces a new size-biased distribution which is partially inspired by [1]. A member of this new class of distributions is shown to be practically significant in modeling real life data. Finally, we ask the reader to investigate further properties and applications of this new class of distributions.

Keywords: Size biased distribution; Regularized beta function; Normal distribution

\section{The Size-Biased Kumaraswamy Distribution}

The concept of weighted distributions is largely credited to Fisher [1,2] and Rao [3]. In the size biased schemes, the probability of sampling an individual is proportional to $X^{r}$ provided that $E_{\theta}\left(X^{r}\right)<\infty$ for all $\theta$. In such situations, the weighted probability density function is defined as

$$
f_{r}(x ; \theta)=\frac{x^{r} f(x ; \theta)}{\mu_{r}^{\prime}}
$$

where $\mu_{r}^{\prime}=\int x^{r} f(x ; \theta) d x$. When the probability of observing a positive-valued random variable is proportional to the value of the variable the resulting distribution is termed size-biased with probability density function

$$
g(x ; \theta)=\frac{x f(x ; \theta)}{\mu_{r}^{\prime}}
$$

The Kumaraswamy distribution [4] has probability density function

$$
g(x ; a, b)=a b x^{a-b}\left(1-x^{a}\right)^{b-1}, 0<x<1 ; a, b>0
$$

and the $\mathrm{r}^{\text {th }}$ order raw moment is given by [1]

$$
\mu_{r}^{\prime}=b B\left(1+\frac{r}{a}, b\right)
$$

where $B\left(1+\frac{r}{a}, b\right)$ is a beta function defined by the integral

$$
B(\alpha, \beta)=\int_{0}^{1} x^{\alpha-1}(1-x)^{\beta-1} d x
$$

Notation 1.1. We write $X \sim \operatorname{SBKD}(a, b)$, if $\mathrm{X}$ is a size biased Kumaraswamy random variable

Proposition 1.2. [1] The PDF of the $\operatorname{SBKD}(a, b)$ distribution is given by

$$
f(x ; a, b)=\frac{a x^{a-1}\left(1-x^{a}\right)^{b-1}}{B\left(1+\frac{1}{a}, b\right)}
$$

where $0<x<1$, and $B\left(1+\frac{r}{a}, b\right)$ is a beta function defined by the integral

with $a, b>0$

$$
B(\alpha, \beta)=\int_{0}^{1} x^{\alpha-1}(1-x)^{\beta-1} d x
$$

Theorem 1.3. [1] The CDF of the $\operatorname{SBKD}(a, b)$ distribution is given by

$$
F(x ; a, b)=I_{x^{a}}\left(1+\frac{1}{a}, b\right)
$$

Where

$$
I_{x^{a}}\left(1+\frac{1}{a}, b\right)==\frac{B\left(x^{a} ; 1+\frac{1}{a}, b\right)}{B\left(1+\frac{1}{a}, b\right)}
$$

is the regularized incomplete beta function and is defined as the ratio of an incomplete beta function,

$$
B(z ; \alpha, \beta)=\int_{0}^{z} x^{\alpha-1}(1-x)^{\beta-1} d x
$$

and the complete beta function, 


$$
B(\alpha, \beta)=\int_{0}^{1} x^{\alpha-1}(1-x)^{\beta-1} d x
$$

with $a, b>0$, and $\mathrm{x} \in(0,1)$

\section{The $\left(\frac{1}{e}\right)^{\alpha}$ PT-G Family of Distributions}

Definition 2.1. [5] Let $\alpha \neq \frac{1}{e}, \alpha>\frac{1}{e}$ and $\xi>0$ where $\alpha$ is the rate scale parameter and is a vector of parameters in the baseline distribution all of whose entries are positive.

A random variable $\mathrm{Z}$ is said to follow the $\left(\frac{1}{e}\right)^{\alpha}$ power transform family of distributions if

the Cumulative Distribution Function (CDF) is given by

$$
F(x ; \alpha, \xi)=\frac{1-e^{-\alpha G(x ; \xi)}}{1-e^{-\alpha}} \quad x \in \mathbb{R},
$$

where the baseline distribution has $\operatorname{CDF} G(x ; \xi)$. Proposition 2.2. [5] The PDF of the $\left(\frac{1}{e}\right)^{\alpha}$ power transform
family distribution is given by

$$
f(x ; \alpha, \xi)=\frac{1}{1-e^{-\alpha}}\left(\alpha g(x ; \xi) e^{-\alpha G(x ; \xi)}\right)
$$

where $\alpha \neq \frac{1}{e}, \alpha>\frac{1}{e}$ and $\xi>0$ and $G(x ; \xi)$ is the baseline cumulative distribution with

probability density function $g(x ; \xi)$

\section{The New Distribution}

By modifying the parameter space for $\alpha$ in the $\left(\frac{1}{e}\right)^{\alpha}$ PT-Standard Uniform family and using it in Theorem 1.3, we have the following

Proposition 3.1. The CDF of the new size biased Kumaraswamy Distribution is given by

$$
\begin{gathered}
F(x ; a, b, \alpha)=I_{\left(\frac{1-e^{-\alpha x}}{1-e^{-\alpha}}\right)^{a}}\left(1+\frac{1}{a}, b\right) \\
I_{\left(\frac{1-e^{-\alpha x}}{1-e^{-\alpha}}\right)^{a}}\left(1+\frac{1}{a}, b\right)=\frac{B\left(\left(\frac{1-e^{-\alpha x}}{1-e^{-\alpha}}\right)^{a} ; 1+\frac{1}{a}, b\right)}{B\left(1+\frac{1}{a}, b\right)}
\end{gathered}
$$

is the regularized incomplete beta function and is defined as the ratio of an incomplete beta function,

$$
B(\alpha, \beta)=\int_{0}^{1} x^{\alpha-1}(1-x)^{\beta-1} d x
$$

and the complete beta function,

$$
B(\alpha, \beta)=\int_{0}^{1} x^{\alpha-1}(1-x)^{\beta-1} d x
$$

with $a, b>0, \alpha \in \mathbb{R}, \alpha \neq 0$, and $\mathrm{x} \in(0,1)$

Using the above, we introduce the following

Definition 3.2. We say $Z$ is a new size-biased Kumaraswamy-G random variable, and write $\mathrm{Z} \sim \operatorname{SBKGD}(\mathrm{a}, \mathrm{b}, \alpha, \xi)$, if the $\operatorname{CDF}$ is given by

$$
F^{*}(x ; a, b, \alpha, \xi):=F(G(x ; \xi), a, b, \alpha)
$$

where $\mathrm{F}$ is given by the previous Proposition, $\mathrm{a}, \mathrm{b}>0, \alpha \in \mathrm{R}, \alpha 6=$ $0, \mathrm{x} \in \mathrm{R}$, and $\xi$ is a vector of parameters in the baseline distribution with $\operatorname{CDF} G(x ; \xi)$

Remark 3.3. The PDF of the new size-biased Kumaraswamy-G family of distributions can be obtained by differentiating the CDF

\section{Practical Illustration}

We assume the baseline distribution is Normal with the following $\mathrm{CDF}$

$$
G(x ; c, d)=\frac{1}{2} \operatorname{erfc}\left(\frac{c-x}{\sqrt{2} d}\right)
$$

where $x, c \in \mathbb{R}, \mathrm{d}>0$, and

$$
\operatorname{erfc}(z)=1-\operatorname{erfc}(z)=1-\frac{2}{\sqrt{\pi}} \int_{0}^{z} e^{-t^{2}} d t
$$

Thus from Definition 3.2 and Proposition 3.1, we have the following

Proposition 4.1. The CDF of the new size-biased KumaraswamyNormal distribution is given by

$$
\left.F^{*}(x ; a, b, \alpha, c, d)=I_{\left(\frac{1-e^{-\frac{1}{2} \alpha e r f\left(\frac{c-x}{\sqrt{2} d}\right)}}{1-e^{-\alpha}}\right)}\right)^{a}\left(\frac{1}{a}+1, b\right)
$$

Where

$$
I_{z}(a, b)=\frac{B(z ; a, b)}{B(a, b)}=\frac{\int_{0}^{z} x^{\alpha-1}(1-x)^{\beta-1} d x}{\int_{0}^{1} x^{\alpha-1}(1-x)^{\beta-1} d x}
$$

with $\mathrm{x}, \mathrm{c} \in \mathbb{R}, \mathrm{d}>0$, and $\operatorname{erfc}(z)=1-\operatorname{erfc}(z)=1-\frac{2}{\sqrt{\pi}} \int_{0}^{z} e^{-t^{2}} d t$

Remark 4.2. We write $Q \sim N$ SBKN D $(a, b, \alpha, c, d)$, if $Q$ is a new size-biased Kumaraswamy-Normal random variable. When we fix a $=1$, we denote the reduced distribution as $\mathrm{N} \operatorname{BIN} \mathrm{D}(\mathrm{b}, \alpha, \mathrm{c}, \mathrm{d})$, and say $\mathrm{Q}$ is a new Beta-I-Normal random variable (Figure 1).

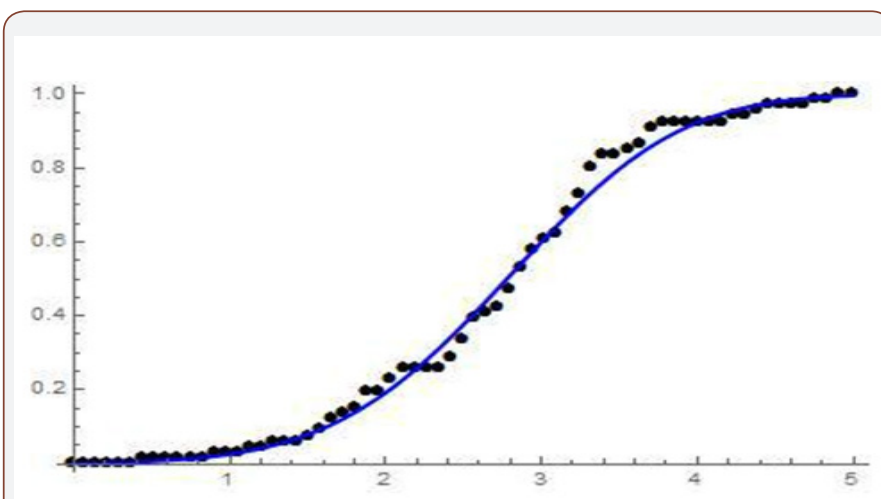

Figure 1: The CDF of COF W $(0.73884,1.47816,0.192653$, 2062.69) fitted to the em-pirical distribution of the data on patients with breast cancer, Section 5.2. 


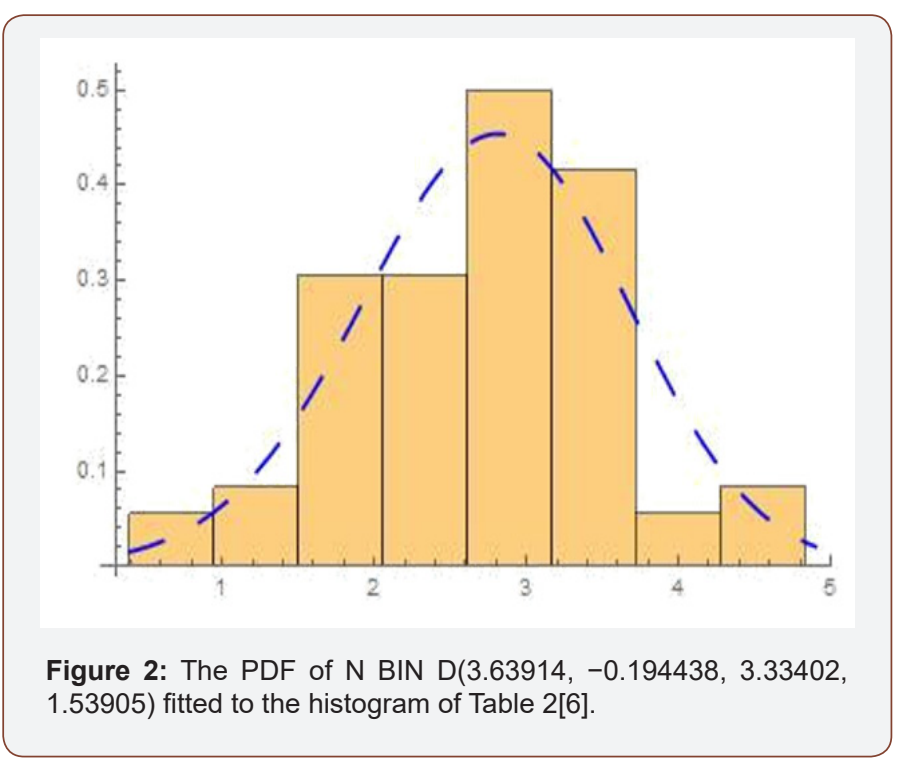

Remark 4.3. The PDF of the new size-biased KumaraswamyNormal distribution can be obtained by differentiating the CDF (Figure 2).

\section{Conclusion}

Size-biased distributions arise naturally in a range of sampling and modeling problems in forestry [7], as such we suggest to the reader to obtain some properties and applications of this new class of distributions as it pertains to forestry.

\section{Acknowledgement}

None.

\section{Conflict of Interest}

No conflict of interest.

\section{References}

1. Dreamlee Sharma, Tapan Kumar Chakrabarty (2016) On Size Biased Kumaraswamy Distribution.

2. Fisher RA (1934) The effects of methods of ascertainment upon the estimation of frequencies. Annals of Eugenics 6(1): 13-25.

3. Rao CR (1965) On discrete distributions arising out of methods of ascertainment. In: Patil GP (Ed.), Classical and Contagious Discrete Distributions. Pergamon Press and Statistical Publishing Society: Calcutta, pp. 320-332

4. Kumaraswamy PA (1980) Generalized probability density function for double-bounded ran-dom processes. Journal of Hydrology 46(1): 79-88.

5. Abdulzeid Yen Anafo (2019) The New Alpha Power Transform: Properties and Ap-plications, Master of Science in Mathematical Sciences Essay, African Institute for Mathematical Sciences (Ghana). Unpublished Manuscript

6. Ayman Alzaatreh, Carl Lee, Felix Famoye (2014) T-normal family of distributions: a new approach to generalize the normal distribution, Journal of Statistical Distributions and Applications 1: 16.

7. Gove JH (2003) Estimation and applications of size-biased distributions in forestry. In: Amaro A, Reed D, Soares P (Eds.), Modeling Forest Systems; CABI Publishing, pp. 201-212. 